\title{
Effect of Lactic Acid Fermentation on Total Phenolic Content and Antioxidant Activity of Fig Fruit Juice (Ficus carica)
}

\author{
Ernanin Dyah Wijayanti*, Nur Candra Eka Setiawan and Jean Patricia Cristi \\ Academy of Pharmacy of Putra Indonesia Malang, Indonesia \\ Jalan Barito Nomor 5, Bunulrejo, Blimbing, Kota Malang, Jawa Timur 65126 \\ *Corresponding author: nanin.wijayanti@gmail.com
}

\begin{abstract}
Background: Fig (Ficus carica) is used for a disease therapy due to its relatively complete nutrition and active compounds as sugar, mineral, vitamin, organic acid and phenolic compounds. A high phenolic compound in fig is related closely to its fruit activity as an antioxidant. Yet, its difficult for phenolic compound to be absorbed by the human body, so a fermentation is required. Objectives: This research aims to determine the effect of lactic acid fermentation on total phenolic content and antioxidant activity of fig fruit juice. Method: Fig fruit juice was fermented at $37^{\circ} \mathrm{C}$ for 24 hours using 4 types of starter bacteria as Lactobacillus acidophilus, L. bulgaricus, L. casei and L. plantarum. The total phenolic content testing was conducted using the spectrophotometry method using sulfanilic acid reagent. The antioxidant activity was tested using the spectrophotometry method using DPPH reagent. Results: The fermentation result of fig fruit juice using Lactobacillus bulgaricus demonstrates the highest increase of total phenolic content $(T P C=0.45 \%)$ and the biggest antioxidant activity (IC50=76.55 ppm) compared to the unfermented ones $($ TPC $=0.09 \%$; IC50=76.7 ppm). The fermentation result of fig fruit juice using other bacteria also demonstrates the increase of total phenolic content with a smaller antioxidant activity compared to the unfermented ones, yet it is still included as a strong activity. Conclusion: Based on the results, it can be concluded that the lactic acid fermentation is able to increase the total phenolic content and preserve the antioxidant activity.
\end{abstract}

Keywords: Antioxidant, Fig, Total Phenolic Content

\section{INTRODUCTION}

Fig fruit is commonly known by the society as a fruit which has many benefits for the health. It is mostly used as an anti-cancer, in degenerative disease therapy, digestion problem, osteoporosis and infectious disease prevention due to its quite complete nutrition contents. That is why, this fruit is known as a "paradise fruit".

Some researchers have demonstrated various fig fruit contents to support its use for the society. Different parts of the plant as fruit, seed, leaf, stem, bud, and sap have various benefits for the health. Dried fig fruit has been known as a source of carbohydrates, sugar, minerals, vitamins, organic acids, and phenolic compounds (Soni et al. 2014).

Fig fruit contains high level of phenolic compounds to support its use as an antioxidant. Yet, mostly he phenolic compounds are difficult to be absorbed by the human body. The highest level of phenolic compounds is found in the vegetables, except flavonol which is found in the glycosylated form and glycosylation is affecting the absorption. To make the absorption easear, the hydrolysis by enzymes or microbes in the digestive tract is 
required (Filannino et al. 2016). In line with the previous statement, the fig fruit contents require the hydrolysis processes, one of them is fermentation process.

Fermentation can increase the nutrition value in the foodstuffs. It can also increase the antioxidant activity in the fermented materials. Fermentation can increase the antioxidative activity by increasing the flavonoid release of plant-based food. Various biochemical changes occurred during the fermentation causing the nutrition component and anti-nutrition ratio changes influencing the product characteristics, as a bioactivity and digestibility (Zhang, Soccol \& Pandey 2012).mThe glycoside conversion into the aglycone by the fermentation is the main principle of the antioxidative activity increase in the plantbased food (Hur et al. 2012).

The most applied fermentation in the foodstuffs is a lactic acid fermentation. This fermentation type uses lactic acid bacteria which safety and benefits are proven. Lactobacillus plantarum is the most used species for the fermentation in the plant-based food products. Other lactic acid bacteria are Lactobacillus acidophilus, Lactobacillus casei, Lactobacillus fermentum, Bifidobacterium animalis subsp. lactis and Bifidobacterium longum (Rodríguez et al. 2009; Marazza et al. 2009). Therefore, Lactobacillus acidophilus, Lactobacillus bulgaricus, Lactobacillus casei and Lactobacillus plantarum are used as a starter in this research. This research aims to determine the effect of lactic acid fermentation on total phenolic content and antioxidant activity of fig fruit juice.

\section{METHOD}

\section{Materials}

Dried fig fruit was purchased from the tin leaf tea producer (Kunta tea) in Gresik, East Java. Lactobacillus acidophillus and Lactobacillus plantarum bacterial cultures were purchased from the Microbiology Laboratory, Agricultural Product Technology Department, Agricultural Technology Faculty, Brawijaya University, Malang, East Java, while Lactobacillus bulgaricus and Lactobacillus casei cultures were isolated from the commercial fermented products.

\section{Fig Fruit Juice Fermentation}

Dried fig fruit was blended and diluted with water with the ratio of 1:5. Then, the obtained juice was filtered to gain the fig fruit juice. The fig fruit juice was pasteurized for 15 minutes at $72^{\circ} \mathrm{C}$, then was left until the temperature dropped to around $40^{\circ} \mathrm{C}$. After that, the juice was added to the bacterial starter $(6 \%)$ and incubated at $37^{\circ} \mathrm{C}$ for 24 hours.

\section{Total Phenolic Content (TPC) Testing}

Some samples were put into the test tube, added with $1 \mathrm{ml}$ of reagent A (Sulfanilic acid $7.64 \%, \mathrm{H}_{2} \mathrm{SO}_{4}$, and Sodium Nitrite $4.8 \%$ ) and $0.5 \mathrm{ml} \mathrm{NaOH} 8 \%$, then shaken and left for 3 minutes to form a color. Then, it was measured for its absorbance using spectrophotometer at $360 \mathrm{~nm}$ of wavelength. The standard curve of phenol with the same procedure was made .

\section{Antioxidant Activity Testing}

Some samples were weighed, dissolved in methanol, added with an absorbent to omit the unwanted compounds. The absorbent contained the mix of $\mathrm{Al}, \mathrm{Mg}, \mathrm{SO}_{4}$, and $\mathrm{Si}$ added with sodium acetate as its buffer. Then, the samples were centrifuged for 10 minutes at $4500 \mathrm{rpm}$ of speed, added with methanol and DPPH, incubated in the dark room, then measured its absorbance. The quercetin standard was made using several concentrations 
(0.5 ppm; 1 ppm; 2 ppm; 4 ppm and 8 ppm), added with methanol and DPPH, incubated in the dark room, measured for its absorbance using spectrophotometer at $517 \mathrm{~nm}$ of wavelength.

\section{RESULT AND DISCUSSION}

\section{Effect of Lactic Acid Fermentation on Phenolic Compounds}

All of the lactic acid bacteria used as starters in this research is able to ferment the fig fruit juice and produce the increase of total phenolic content significantly. Before fermented, the total phenolic content of fig fruit juice was $0.09 \%$, while after fermented, the total phenolic content increase between $100 \%$ to $400 \%$. The highest increase was obtained from the fermentation result using Lactobacillus bulgaricus producing the total phenolic content of $0.45 \%$. The fermentation using L. plantarum producing the total phenolic content of $0.33 \%$, using L. acidophilus producing the total phenolic content of $0.21 \%$, and using L. casei producing the total phenolic content of $0.19 \%$ (Figure 1).

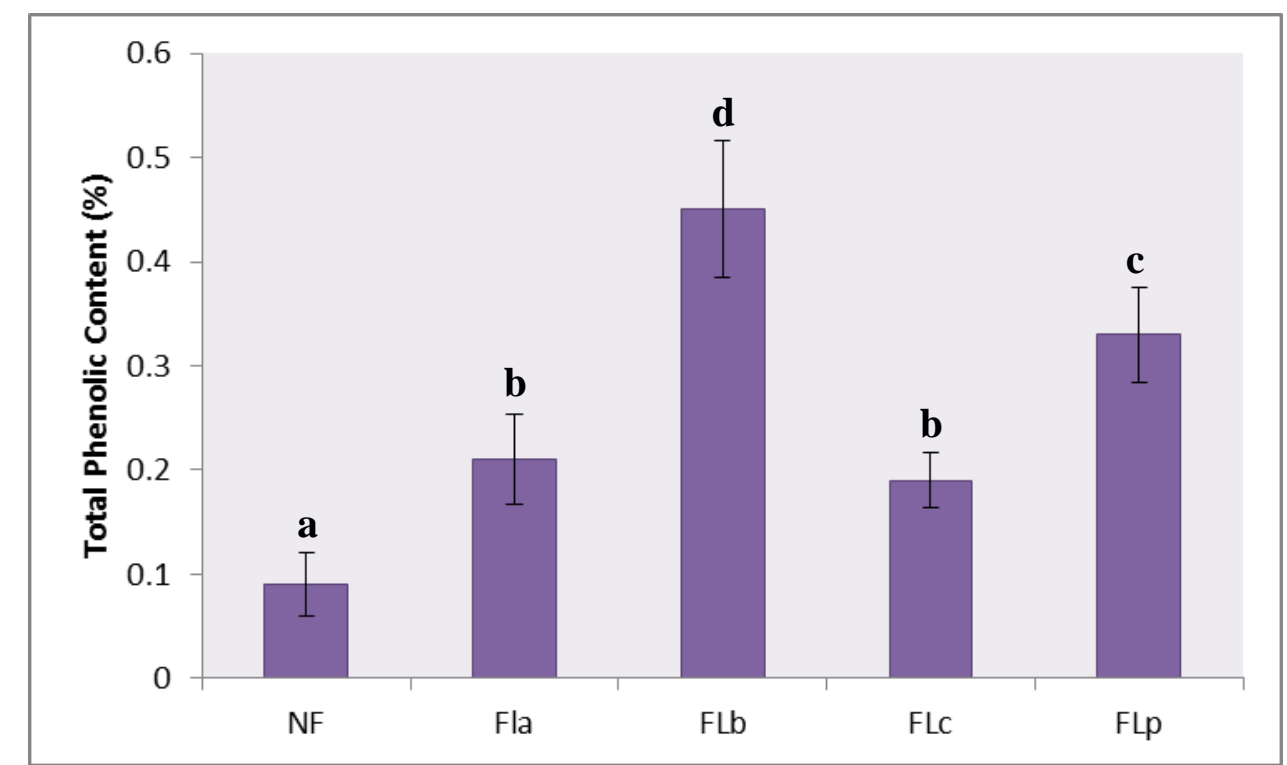

Figure 1 Total Phenolic Content of Fig Fruit Juices. NF=Non Fermented; Fla= Fermented with Lactobacillus acidophillus; FLb= Fermented with Lactobacillus bulgaricus; FLc= Fermented with Lactobacillus casei; FLp= Fermented with Lactobacillus plantarum. The different alphabetical notations show the significant differences based on the Tukey HSD testing with the confidence interval of $95 \%$.

The phenolic compounds were known as a secondary metabolite, which is important, coming from the phenylalanine and tyrosine. These compounds contain in a large amount variously in plants. Plant phenolic compounds are important because their hydroxyl group can prevent the free radicals. Plant materials containing phenolic compounds are increasingly used in the food industries because they can prevent the oxidative degradation in fat and increase the quality and nutritional value of food (Nithya et al. 2016).

Based on the total phenolic content determination result of fig fruit juice, the increase of total phenolic content after the juice experiencing the lactic acid fermentation can be determined. It shows that the lactic acid fermentation can increase the total phenolic content of fig fruit juice. The increase of total phenolic content can occur due to the lactic acid bacteria activity used as starters in the fermentation process. It was stated that the fermentation process causes the microbial enzyme release producing higher chemical compounds from the plant as flavonoids, tannin, alkaloids, and phenylpropanoid (Nazarni 
et al. 2016). The lactic acid bacteria existence in the fermentation contributes to the simple phenolic conversion and phenolic compounds depolymerization with the high molecular weight (Othman et al. 2009).

The increase of total phenolic content in the fermentation result is caused by the enzymatical reaction in the substrate, so it releases rather high phenolic compounds as a final product. The natural fermentation using microorganisms stimulates the $\mathrm{pH}$ reduction so that several involved enzymes in the complex polyphenols hydrolysis were activated resulting on the active, simpler and higher polyphenols. During the fermentation, the $\beta$ glucosidase from the microbes can hydrolyze phenolic and flavonoid. L. plantarum was reported to have a strong glucosidase activity. Thus, the active substance experiencing increase is expected to be converted from the enzymatic cleavage of corresponding glucosides (Dueñas et al, 2005). It was also stated that the fermentation can induce the cell wall structural breakdown causing the bioactive compounds release and/or synthesis. During the fermentation, the bound phenol can be released enzymatically (Zhang, Soccol \& Pandey 2012).

The increase of total phenolic compounds in the fermentation of fig fruit juice is in accordance with some of the reported studies as the increase of total phenolic content on jaruk tigarun flowers (Nazarni et al. 2016), Graptopetalum paraguayense E. Walther (Wu, Su \& Cheng 2011), Echinacea spp. (Rizzello \& Coda 2013), and Malaysian herbal teas (Ibrahim, Mustafa \& Ismail 2014). It was also reported that parts of plants experiencing the increase of total phenolic content after the fermentation ( $\mathrm{Ng}$ et al. 2014).

\section{Effect of Lactic Acid Fermentation on Antioxidant Activity}

The lactic acid fermentation produces a various antioxidant activity of the fig fruit juice. The antioxidant activity was demonstrated by the IC50 value. IC50 or inhibition concentration 50 is an antioxidant concentration required to inhibit $50 \%$ of free radicals. The higher of IC50 value means the lower of antioxidant activity. IC50 is defined as the antioxidant amount required for reducing 50\% of DPPH absorbance from the early absorbance (Mishra, Ojha \& Chaudhury 2012).

Before fermented, the fig fruit juice showed $76.7 \mathrm{ppm}$ of IC50 value. The fermentation using L. bulgaricus shows the increase of antioxidant activity with the IC50 value of $76.55 \mathrm{ppm}$, yet the increase is not significant. The fermentation using $L$. case $i$ shows the reduction of antioxidant activity with the IC50 value of $77.41 \mathrm{ppm}$, yet the reduction is not significant too. The fermentation using L. acidophillus and using $L$. plantarum produce the significant reduction of antioxidant activity with the IC50 value of $105.42 \mathrm{ppm}$ and $95.94 \mathrm{ppm}$. In conclusion, the lactic acid fermentation can affect the antioxidant activity of fig fruit juice.

If observed from its IC50 value, the fermentation using L. bulgaricus, L. casei, and $L$. plantarum produces the active antioxidant activity, whereas the fermentation using $L$. acidophillus produces the average antioxidant activity. It refers to antioxidant activity power which is determined by the IC50 value, where less than $50 \mathrm{ppm}$ classified as strong, 50-100 ppm classified as active, 100-250 ppm classified as average, 250-500 ppm classified as weak and more than 500 ppm classified as inactive (Salusu et al. 2017).

The antioxidant activity change after the fermentation process can be affected by the starter bacteria used. It was stated that the fermentation can affect the total phenolic content and antioxidative activity with the level of influence depending on the species of microorganisms used. The fermentation process is supposed to increase the antioxidant activity (Zhang, Soccol \& Pandey 2012). The lactic acid bacteria with the $\beta$-glucosidase activity (including Lactobacillus acidophilus, L. casei, L. plantarum, L. fermentum, 
Bifidobacterium animalis subsp. lactis and Bifidobacterium longum) can increase the aglycone during the fermentation. The aglycone acts as an antioxidant (Marazza et al. 2009). The finding shows that $L$. bulgaricus and $L$. casei can produce the fermented fig fruit juice by retaining the antioxidant activity demonstrated by the insignificantly different IC50 value. Nevertheless, $L$. acidophillus and $L$. plantarum produce the fermented fig fruit juice with a lower antioxidant activity compared to the activity before the fermentation. The exact same thing also happens in the Chetoui olives (Olea europaea L.) fermentation with the reduction of antioxidant activity approximately between $50 \%$ and $72 \%$ (Othman et al. 2009).

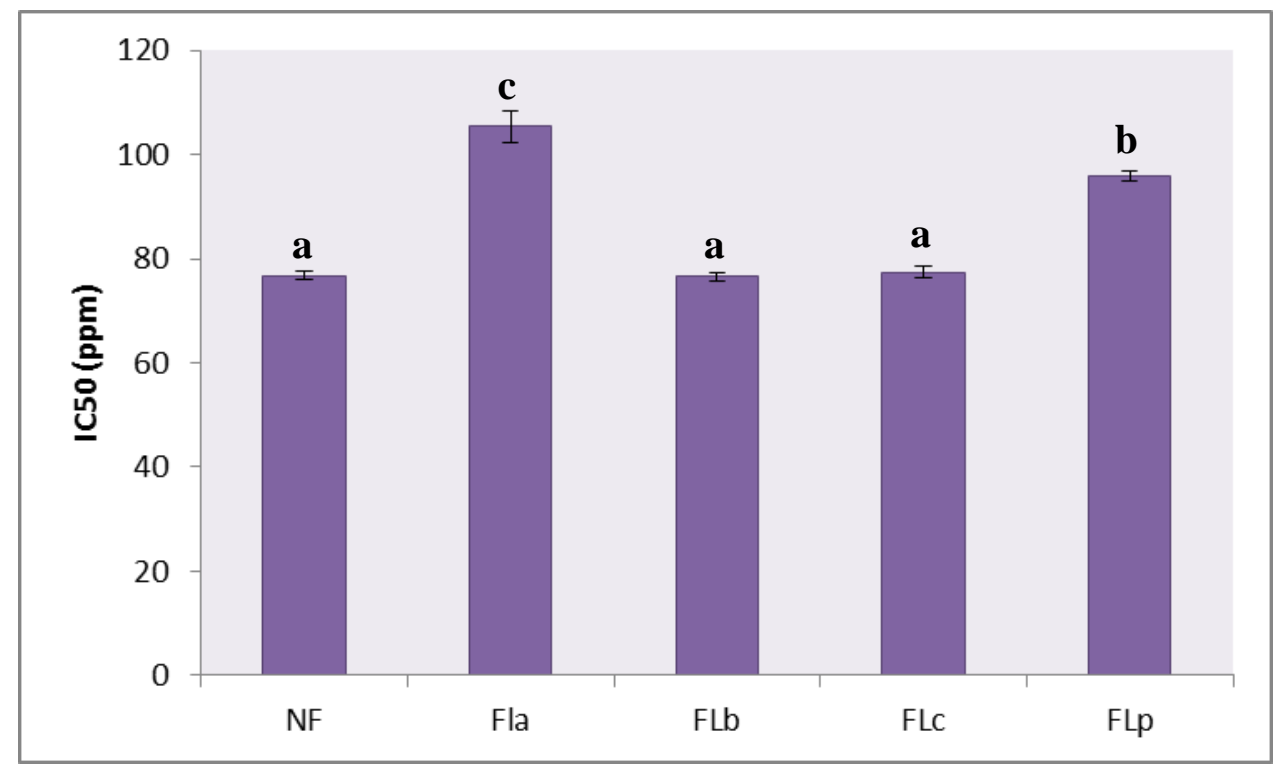

Figure 2 IC50 of Fig Fruit Juice. NF=Non Fermented; Fla= Fermented with Lactobacillus acidophillus; FLb= Fermented with Lactobacillus bulgaricus; FLc $=$ Fermented with Lactobacillus casei; FLp= Fermented with Lactobacillus plantarum. The different alphabetical notations show the significant differences based on the Tukey HSD testing with the confidence interval of $95 \%$.

The antioxidant activity of fig fruits juice can be produced by the secondary metabolite compounds in fig fruit. There was a research stated that in the dried fig fruit there are phenolic (10.90 $\mu \mathrm{g} \mathrm{GAE} / \mathrm{mg}$ sample), flavonoid $(2.75 \mu \mathrm{g} \mathrm{CE} / \mathrm{mg}$ sample), the alkaloid (9.6\%) and saponin (0.59\%) (Aksoy et al. 2013). Generally, there is an antioxidant compound in the form of phenolic. The antioxidant characteristics of phenolic compounds come from the proton-releasing ability, chelate formation, and radical dismutation. The phenolic compounds give the hydrogen atom from hydroxyl group to the radical and form a stable phenoxyl radical so it plays a significant role in the antioxidant activity. Therefore, the determination of phenolic total content is very important to determine the antioxidant ability of plant extracts (Aksoy et al. 2013).

The increase of phenolic compounds is supposed to produce a higher antioxidant activity since there is more hydroxyl group capable of preventing the free radicals. Yet, in this research, the antioxidant activity produced by the fermented fig fruit juice does not experience any increase as occurred in the total phenolic content. It shows that the hydroxyl group number of phenolic compounds is not the only factor determining the antioxidant activity.

Some studies state that the antioxidant activity structure of flavonoid and phenolic acid depending on the hydroxyl group position of phenolic and the existence of other functional groups on the whole molecule as double bond and its conjugation towards the 
hydroxyl and ketone groups (Mishra et al. 2012). Several studies indeed show the positive correlation between the antioxidant activity and total phenolic content, as in the antioxidant activity of Thermopsis turcica (Aksoy et al. 2013), buckwheat (Fagopyrum esculentum Moench) (Sun \& Ho 2005), ulam raja' (Cosmos caudatus), 'kesum' (Polygonum minus), 'selom' (Oenanthe javanica), 'pegaga' (Centella asiatica) and 'curry leaf' (Murraya koenigii) (Faujan et al. 2009), Radix Angelicae Sinensisis (Li et al. 2009), and Solanum tuberosum (Hesam et al. 2012). Yet, the other studies demonstrate the contrast result showing the negative correlation between the antioxidant activity and total phenolic content, as in the antioxidant potency of Chenopodium quinoa and Amaranthus spp seeds obtained by using 3 different methods (FRAP, DPPH and carotene bleaching) that negatively correlates with the total phenolic content (Nsimba et al. 2008). The negative correlation was also found in the antioxidant activity and total phenolic content of 15 genotypes of selected Zizyphus jujube Mill. from Turkey (Kamiloglu et al. 2009). Another study also demonstrated the negative correlation between the antioxidant activity and total phenolic content of ethanolic extract of selected plants. The ethanolic extract of Euodia redlevi contains the highest phenolic compounds compared to the phenolic compounds of other extracts. The lowest total phenolic content was demonstrated by the extract of Centella asiatica, yet it produces the second highest antioxidant activity, both in the DPPH and SOD methods (Rafat, Philip \& Muniandy 2010).

Based on the previous statements, there is a possibility causing the reduction of antioxidant activity of fermented fig fruit juice although there is an increase of total phenolic content. First, there is a change in the structure of hydroxyl group of phenolic compounds of fig fruit after the fermentation process, so it does not produce the increase of antioxidant activity even when there is an increase of total phenolic content. Another possibility is the non-phenolic compound existence of fig fruit having a role in the antioxidant activity ability.

\section{CONCLUSION}

The lactic acid fermentation is able to increase the total phenolic content significantly and preserve the antioxidant activity, which is classified as a active category. The increase of the total phenolic content of fig fruit juice after the fermentation is not followed by the antioxidant activity increase. It is due to the hydroxyl group structure change or the non-phenolic compound existence acting as an antioxidant.

\section{ACKNOWLEDGEMENT}

Acknowledgment is dedicated to the Directorate of Research and Community Service - the Directorate of General of Research and Development Reinforcement - the Ministry of Research, Technology and Higher Education of the Republic of Indonesia that has funded this research through the Novice Lecturer Research Grant Program.

\section{REFERENCES}

Aksoy, L., E. Kolay, Y. Agılonu, Z. Aslan, M. Kargioglu. (2013). Free Radical Scavenging Activity, Total Phenolic Content, Total Antioxidant Status, and Total Oxidant Status of Endemic Thermopsis turcica. Saudi Journal of Biological Sciences 20, 235-239.

Dueñas, M., Fernández, D., Hernández, T., Estrella, I. and Muñoz, R. (2005). Bioactive phenolic compounds of cowpeas (Vigna sinensis L.). Modifications by fermentation 
with natural microflora and with Lactobacillus plantarum ATCC 14917. Journal of The Science of Food and Agriculture 85 (2): 297-304.

Faujan, N.H., Noriham, A., Norrakiah, A.S., Babji, A.S., (2009). Antioxidant Activity Of Plants Methanolic Extracts Containing Phenolic Compounds. African Journal of Biotechnology 8: 484-489.

Filannino, P., I. Cavoski, N. Thlien, O. Vincentini, M. De Angelis, M. Silano, M. Gobbetti1, R. Di Cagno. (2016). Lactic Acid Fermentation of Cactus Cladodes (Opuntia ficus-indica L.) Generates Flavonoid Derivatives with Antioxidant and Anti-Inflammatory Properties. Plos One, DOI:10.1371/journal.pone.0152575.

Hur, S. J., S. Y. Lee, Y. Kim, I. Choi, G. Kim. (2014). Effect of Fermentation on The Antioxidant Activity in Plant-Based Foods. Food Chemistry 160: 346-356.

Hesam, F., G. R. Balali, R. T. Tehrani. (2012). Evaluation Of Antioxidant Activity Of Three Common Potato (Solanum tuberosum) Cultivars in Iran. Avicenna Journal of Phytomedicine Vol. 2, No. 2: 79-85.

Ibrahim, N. A., S. Mustafa and A. Ismail. (2014). Effect of Lactic Fermentation on The Antioxidant Capacity of Malaysian Herbal Teas. International Food Research Journal 21(4): 1483-1488.

Kamiloglu, O., S. Ercisli, M. Sengül, C. Toplu and S. Serçe. (2009). Total Phenolics And Antioxidant Activity Of Jujube (Zizyphus jujube Mill.) Genotypes Selected from Turkey. African Journal of Biotechnology Vol. 8 (2): 303-307.

Li, X., Wu, X., Huang, L., (2009). Correlation Between Antioxidant Activities And Phenolic Contents Of Radix Angelicae Sinensis (Danggui). Molecules 14, 53495361.

Marazza, J. A., M. S. Garro, G. S. de Giori. (2009). Aglycone production by Lactobacillus rhamnosus CRL981 during soymilk fermentation. Food Microbiology 26: 333-339.

Mishra, K, H. Ojha, N. K. Chaudhury. (2012). Estimation Of Antiradical Properties Of Antioxidants Using DPPH Assay: A Critical Review And Results. Food Chemistry 130: 1036-1043.

Nazarni, R., Purnama, D., Umar, S. and Eni, H. (2016). The Effect Of Fermentation On Total Phenolic, Flavonoid And Tannin Content And Its Relation To Antibacterial Activity In Jaruk Tigarun (Crataeva nurvala, Buch HAM). International Food Research Journal 23 (1): 309-315.

Nithya, T.G, Jayanthi J, Ragunathan M.G. (2016). Antioxidant Activity, Total Phenol, Flavonoid, Alkaloid, Tannin, And Saponin Contents Of Leaf Extracts Of Salvinia Molesta D. S. Mitchell (1972). Asian Journal of Pharmaceutical and Clinical Research, Vol 9, Issue 1, 200-203.

Ng, C. C., Wang, C. Y., Wang, Y. P., Tzeng, W. S. and Shyu, Y. T. (2011). Lactic Acid Bacterial Fermentation On The Production Of Functional Antioxidant Herbal Anoectochilus formosanus Hayata. Journal of Bioscience and Bioengineering 111(3): 289-293.

Nsimba, R. Y., H. Kikuzaki, Y. Konishi. (2008). Antioxidant Activity Of Various Extracts And Fractions Of Chenopodium quinoa and Amaranthus spp. Seeds. Food Chemistry 106: 760-766.

Othman, N. B., D. Roblain, N. Chammen, P. Thonart, M. Hamidi. (2009). Antioxidant Phenolic Compound Loss During The Fermentation of Chetoui Olives. Food Chemistry 116: 662-669.

Rafat, A., K. Philip, S. Muniandy. (2010). Antioxidant Potential And Phenolic Content Of Ethanolic Extract Of Selected Malaysian Plants. Research Journal of Biotechnology Vol. 5 (1) : 16-19. 
Rizzello, C. G. and Coda, R. (2013). Lactic Fermentation As A Tool To Enhance The Functional Features of Echinacea spp. Microbial Cell Factory 12 (44): 1-15.

Rodríguez, H., B. de Las Rivas, F. L. de Felipe Toledano, J. M. Mancheño. (2009). Food Phenolics And Lactic Acid Bacteria. International Journal of Food Microbiology 132: 79-90.

Salusu, H. D., F. Ariani, E. Obeth, M. Rayment, E. Budiarso, I. W. Kusuma and E. T. Arung. (2017). Phytochemical Screening and Antioxidant Activity of Selekop (Lepisanthes amoena) Fruit. AGRIVITA Journal of Agricultural Science, 39 (2): $214-$ 218.

Sun, T., Ho, C.T., (2005). Antioxidant Activities Of Buckwheat Extracts. Food Chemistry. 90: 743-749.

Soni, N., S. Mehta, G. Satpathy, R. K Gupta. (2014). Estimation of Nutritional, Phytochemical, Antioxidant And Antibacterial Activity of Dried Fig (Ficus carica). Journal of Pharmacognosy and Phytochemistry; 3 (2): 158-165.

Wu, S., Y. Su, H. Cheng. (2011). Antioxidant Properties of Lactobacillus-Fermented And Non-Fermented Graptopetalum Paraguayense E. Walther at Different Stages Of Maturity. Food Chemistry.

Zhang, Z., C. Soccol, A. Pandey. (2012). Production of Powerful Antioxidant Supplements via Solid-State Fermentation of Wheat (Triticum aestivum Linn.). Food Technology and Biotechnology ISSN 1330-9862. 50 (1) 32-39. 